\title{
Acknowledgement to Reviewers of Hydrology in 2017
}

\author{
Hydrology Editorial Office \\ MDPI AG, St. Alban-Anlage 66, 4052 Basel, Switzerland; hydrology@mdpi.com
}

Received: 9 January 2018; Accepted: 9 January 2018; Published: 9 January 2018

Peer review is an essential part in the publication process, ensuring that Hydrology maintains high quality standards for its published papers. In 2017, a total of 62 papers were published in the journal. Thanks to the cooperation of our reviewers, the median time to first decision was 18 days and the median time to publication was 47 days. The editors would like to express their sincere gratitude to the following reviewers for their time and dedication in 2017:

\author{
Aa, Geruo \\ Abo, Rudy K. \\ Afzal, Muhammad \\ Ahilan, Sangaralingam \\ Albano, Raffaele \\ Al-Faraj, Furat \\ Al-Yaari, Amen \\ Amatya, Devendra \\ Antunes, Isabel Margarida Horta Ribeiro \\ Apollonio, Ciro \\ Assani, Ali \\ Ayele, Gebiaw T. \\ Balling, Robert \\ Bao, Jie \\ Barbetta, Silvia \\ Barfus, Klemens \\ Becker, Bernhard P. J. \\ Benjankar, Rohan \\ Beranová, Romana \\ Bermúdez, María \\ Bhattarai, Nishan \\ Bhattarai, Rabin \\ Binet, Stéphane \\ Boschetti, Tiziano \\ Bren, Leon \\ Burri, Nicole \\ Caloiero, Tommaso \\ Cave, Rachel \\ Chanudet, Vincent \\ Chattopadhyay, Somsubhra \\ Chen, Jung-Wei \\ Chen, $\mathrm{Lu}$ \\ Chen, Wei-Bo \\ Chun, Kwok Pan \\ Chun, Sejong
}

Comarazamy, Daniel

Conyers, Lawrence B.

Curtis, Chris J.

Dąbek, Paweł

Darvini, Giovanna

Dawes, Warrick R.

De Luca, Davide Luciano

De Schepper, Guillaume

Deb, Proloy

Dehkordi, S. Emad

Di Paola, Francesco

Diekkrüger, Bernd

Dimas, A. A.

Dimitriou, Elias

Diomede, Tommaso

Djebou, Dagbegnon C. Sohoulande

Du, Xinzhong

Dunn, Ryan J. K.

Duque, Carlos

Esser, Bradley K.

Ganora, Daniele

Gao, Peng

Garcia, Pilar

Garcia, Ricardo

Geng, Xiaolong

Giglioni, Michele

Gioia, Andrea

Gopinath, Girish

Gorgoglione, Angela

Gourbesville, Philippe

Hanson, Adrian

Hapsari, Ratih Indri

Hassanzadeh, Elmira

$\mathrm{He}$, Minxue

He, Xiaogang 
Henry, Tiernan

Hiraishi, Tetsuya

Hrachowitz, Markus

Jakab, Gergely

Jaramillo, Fernando

Jiang, Peng

Jones, Peter

Kahler, David

Kamali, Bahareh

Kannan, Narayanan

Kelly, Jacque

Kim, Mingu

Kim, Sang Ug

Klonner, Carolin

Kochanek, Krzysztof

Kumar, Parveen

Kwok-wing, Chau

Lai, Wencong

Larsen, Morten Andreas Dahl

Laurien, Eckart

Lee, Jeongwoo

Lee, Se-Yeun

Lei, TingWu

Lensky, Nadav

Li, Yuan

Linhoff, Benjamin

Liolios, Konstantinos A.

Lira, Cristina

Liu, Chunlu

Liu, Tie

Lo, Kwong-Fai Andrew

Loaiciga, Hugo A.

Lotsari, Eliisa

Luetkemeier, Robert

Luís, Ana

Ma, Chunfeng

$\mathrm{Ma}, \mathrm{Wu}$

MACA, Petr

Magdaleno, Fernando

Manuela Portela, Maria

Marcinkowski, Pawel

Masseroni, Daniele

Mateo-Lázaro, Jesús

Mbanguka, Rene

McGrane, Scott

Mendoza, Rose Marie O.

Michaelsen, Joel

Miyamoto, Hitoshi

Möck, Christian

Morales-Marin, Luis

Morsy, Mohamed

Musa, Zahrah N
Najibi, Nasser

Napolitano, Francesco

Nardi, Fernando

Nazeer, Majid

Noori, Navideh

Oehler, Till

Omani, Nina

Orlowski, Natalie

Osinska-Skotak, Katarzyna

Page, Declan

Panagopoulos, Yiannis

Panagoulia, Dionysia

Papastergiadou, Eva

Paul, George

Pelletier, Mathew

Pérez-Sánchez, Julio

Polo, María José

Pradhanang, Soni M.

Qiao, Lei

Quaranta, Emanuele

Radice, Alessio

Rafiei Emam, Ammar

Rajib, Adnan

Ravazzani, Giovanni

Redwan, Mostafa

Rees, Simon

Rivera, Diego

Rodrigo-Comino, Jesús

Rojas, Rodrigo

Romatschke, Ulrike

Ryżak, Magdalena

Saavedra, Oliver C.

Saha, Gopal Chandra

Saifullah, Muhammad

Samela, Caterina

Santos, José Maria

Sappa, Giuseppe

Sawada, Yohei

Scarfato, Maddalena

Seki, Katsutoshi

Seo, Youngmin

Shahabfar, Alireza

Shalaby, Ahlam I.

Shaw, Glenn D.

Shen, Shuilong

Škrinár, Andrej

Song, Lixiang

Spiliotopoulos, Marios

Sprigg, William A

Sraj, Mojca

Stacke, Tobias

Stathis, Dimitrios 
Steenhuis, Tammo

Stefanidis, Kostas

Steidl, Joerg

Sy, Ibrahima

Szypłowska, Agnieszka

Tallini, Marco

Tazioli, Alberto

Thejll, Peter

Tolika, Konstantia

Valdinoci, Enrico

Valipour, Mohammad

Volpi, Elena

Wan, Zhanming
Wang, Xixi

Waugh, William

Wilczek, Andrzej

Williams, Charles

Wojtkowska, Małgorzata

$\mathrm{Xu}$, Tianfang

Xu, Ye-Shuang

Yan, Hongxiang

Yang, Jiachuan

Yin, Zhenliang

Zarrella, Angelo

Zischg, Andreas

(C) 2018 by the authors; licensee MDPI, Basel, Switzerland. This article is an open access article distributed under the terms and conditions of the Creative Commons Attribution (CC-BY) license (http://creativecommons.org/licenses/by/4.0/). 